\title{
Word-Sense Disambiguation Using Statistical Models of Roget's Categories Trained on Large Corpora
}

\author{
David Yarowsky
}

\author{
AT\&T Bell Laboratories \\ 600 Mountain Avenue \\ Murray Hill NJ, 07974 \\ yarowsky@research.att.com
}

\begin{abstract}
This paper describes a program that disambiguates English word senses in unrestricted text using statistical models of the major Roget's Thesaurus categories. Roget's categories serve as approximations of conceptual classes. The categories listed for a word in Roget's index tend to correspond to sense distinctions; thus selecting the most likely category provides a useful level of sense disambiguation. The selection of categories is accomplished by identifying and weighting words that are indicative of each category when seen in context, using a Bayesian theoretical framework.
\end{abstract}

Other statistical approaches have required special corpora or hand-labeled training examples for much of the lexicon. Our use of class models overcomes this knowledge acquisition bouleneck, enabling training on unrestricted monolingual text without human intervention. Applied to the 10 million word Grolier's Encyclopedia, the system correctly disambiguated $92 \%$ of the instances of 12 polysemous words that have been previously sudied in the literature.

\section{Problem Formulation}

This paper presents an approach to word sense disambiguation that uses classes of words to derive models useful for disambiguating individual words in context. "Sense" is not a well defined concept; it has been based on subjective and often subtle distinctions in topic, register, dialect, collocation, part of speech and valency. For the purposes of this study, we will define the senses of a word as the categories listed for that word in Roget's Intemational Thesaurus (Fourth Edition Chapman, 1977). ${ }^{1}$ Sense disambiguation will constitute

1. Note that this edition of Roget's Thesaurus is much more extensive than the 1911 version, though comewhat more difficult to obtuin in electronic form. One could use other other concept hienarchies, wheh an WordNer (Miller, 1990) or the LDOCE abject codes (Slator, 1991). All that is necessary is a set of semantic calegories and a his of the words in each calegory. selecting the listed category which is most probable given the surrounding context. This may appear to be a particularly crude approximation, but as shown in the example below and in the table of results, it is surprisingly successful.

\begin{tabular}{|c|c|}
\hline Input & Output \\
\hline $\begin{array}{l}\text { Treadmills attached to cranes were uned to lift henvy } \\
\text { for supplying power for cranes, hoists, and lifts. } \\
\text { bowe this height, tower crane is often uned SB This } \\
\text { elaborate countship rituals cranes build a nest of vegetati } \\
\text { are more closely related to cranes and rails.SB They ran } \\
\text { low trees .PP At least five crane tpecies are in danger of }\end{array}$ & $\begin{array}{l}\text { TOOLS } \\
\text { TOOLS } \\
\text { TOOLS } \\
\text { ANIMAL } \\
\text { ANIMAL } \\
\text { ANIMAL. }\end{array}$ \\
\hline
\end{tabular}

Not only do the Roget categories succeed in partitioning the major senses, but the sense tags they provide as output are far more mnemonic than a dictionary numbering such as "crane 1.2". Should such a dictionary sense number be desired as output, section 5 will outline how a linkage between Roget categories and dictionary definitions can be made.

We will also focus on sense distinctions within a given part of speech. Distinctions between parts of speech, should be based on local syntactic evidence. We use a stochastic part-of-speech tagger (Church, 1989) for this purpose, nun as a preprocessor.

\section{Proposed Method}

The strategy propased here is based on the following three observations: 1) Different conceptual classes of words, such as ANIMALS or MACHINES tend to appear in recognizably different contexts. 2) Different word senses tend to belong to different conceptual classes (crane can be an ANIMAL or a MACHINE). 3) If one can build a context discriminator for the conceptual classes, one has effectively built a context discriminator for the word senses that are members of those classes. Furthermore, the context indicators for a Roget category (e.g. gear, piston and engine for the category TOOLS/MACHINERY) will also tend to be context indicators for the members of that category (such as the machinery sense of crane). 
We atuempt to identify, weight and utilize these indicative words as follows. For each of the 1042 Roget Categories:

1. Collect contexts which are representative of the Roget category

2. Identify salient words in the collective context and determine weights for each word, and

3. Use the resulting weights to predict the appropriate category for a polysemous word occurring in novel text.

\subsection{Step 1: Collect Contexts which are Representative of the Roget category}

The goal of this step is to collect a set of words that are typically found in the context of a Roget category. To do this, we extract concordances of 100 surrounding words for each occurrence of each member of the category in the corpus. Below is a sample set of partial concordances for words in the category TOOLS/MACHINERY (348). The complete set contains 30,924 lines, selected from the particular training corpus used in this study, the 10 million word, Jume 1991 electronic version of Grolier's Encyclopedia.

CARVING .SB The gutuer adz has a concave blade for fonis uipment such as a hydraulic shovel capable of lifting 26 cubic on .SB Resembling a power shovel mounted on a floating lut ujpment, valves for nuclear generators , oil-refinery turbines $00 \mathrm{BC}$, flint-edged wooden sickles were used to gather wikl 1-penetrating carbide-tipged drills forced manufacturers to fi ent heightens the colors .SB Drills live in the forests of equa traditional ABC method and drill were unchanged, and dissa nter of rotation .PP A tower crane is an assembly of fabricat rshy areas .SB The crowned crane, however, occasionally

For optimal training, the concordance set should only include references to the given category. But in practice it will unavoidably include spurious examples since mauy of the words are polysemous (such as drill and crane in lines 7,8 , and 10 above).

While the level of noise introfuced through polysemy is substantial, it can usually be tolerated because the spurious senses are distributed through the 1041 other categories, whereas the signal is concentrated in just onc. Only if several words had secondary senses in the samc category would context typical for the other category appear significant in this context.

However, if one of these spurious senses was frequent and dominated the set of examples, the situation could be disastrous. An attempt is made to weight the concordance data to minimize this effect and to make the sample representative of all tools and machinery, not just the more common ones. If a word such as drill occurs $k$ tinies in the conpus, all words in the context of drill contribute weight $1 / k$ to frequency sums.

Despite its flaws, this weighted matrix will serve as a representative, albeit noisy, sample of the typical context of TOOLS/MACHINERY in Grolier's encyclopedia.

\subsection{Step 2: Identify salient words in the collective context, and weight appropriately}

Intuitively, a salient wond ${ }^{2}$ is one which appears significantly more often in the context of a category than at other points in the corpus, and hence is a better than average indicator for the category. We formalize this with a mutual-information-like estimate: $\operatorname{Pr}(w \mid R C a t) / \operatorname{Pr}(w)$, the probability of a word (w) appearing in the contexl of a Roget category divided by its overall probability in the corpus.

It is important to exercise some care in estimating $\operatorname{Pr}(w \mid R C a l)$. In principle, one could simply count the number of times that $w$ appears in the collective context. However, this estimate, which is known as the maximum likelihood estimate (MLE), can be unreliable, especially when $w$ does not appear very often in the collective context. We have smoothed the local estimates of $\operatorname{Pr}(w \mid R C a t)$ with global estimates of $\operatorname{Pr}(w)$ to obtain a more reliable estimatc. Estinates obtained from the local context are subject to measurement crrors whereas estimates oblained from the global context are subject to being irrelevant. By interpolating between the two, we attempt to find a compromise between the two sources of error. This procedure is based on recent work pioneered by William Galc, and is explaincd in detail in another paper (Gale, Church and Yarowsky, 1992). Space does not permit a complete description here.

Below are snilient words for Roget catcgorics 348 and 414. Those selected are the most important to the nodels, where importance is defined as the product of salience and local frequency. That is to say important words are distinctive and frequent.

The numbers in parentheses are the log of the salience $(\log \operatorname{Pr}(w \mid R C a t) / \operatorname{Pr}(w))$, which we will henceforth refer to as the word's weight in the statistical model of the category.

2. J'or illustrative simplicity, we will refer to words in context. In practice, all operations are aciualiy performed on the lemmas of the words (eal/V = eat,cats,cating, atc, eaten), and inflectiond dianctions are ignorred. While this achieves more concentrated end better estimated stalistics, it throws awny useful information which may be exphoited in future work 


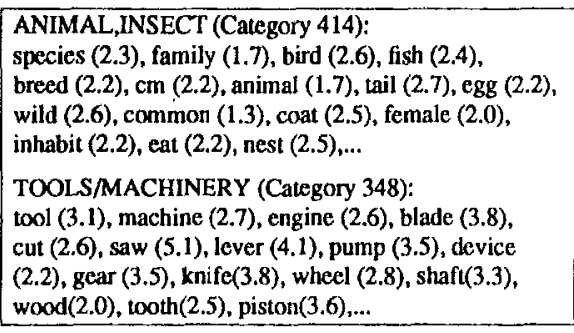

Notice that these are not a list of members of the category; they are the words which are likely to co-occur with the members of the category. The complete list for TOOLSMACHINERY includes a broad set of relations, such as meronomy (blade, engine, gear, wheel, shaft, tooth. piston and cylinder), typical functions of machines (cut, rotate, move, turn, pull), typical objects of those actions (wood, metal), as well as typical modifiers for machines (electric, mechanical, pneumatic). The list for a category typically contains over 3000 words, and is far richer than can be derived from a dictionary definition.

\subsection{Step 3: Use the resulting weights to predict the appropriate category for a word in novel text}

When any of the salient words derived in step 2 appear in the context of an ambiguous word, there is evidence that the word belongs to the indicated category. If several such words appear, the evidence is compounded. Using Bayes' rule, we sum their weights, over all words in context, and determine the category for which the sum is greatest $^{3}$.

$$
\underset{R C a t}{A R G M A X} \sum_{w \text { it context }} \log \frac{\operatorname{Pr}(w \mid R C a t) \times \operatorname{Pr}(R C a t)}{\operatorname{Pr}(w)}
$$

The context is defined to extend 50 words to the left and 50 words to the right of the polysemous word. This range was shown by Gale, Church and Yarowsky (1992) to be useful for this type of broad topic classification, in contrast to the relatively narrow ( $\pm 3-6$ word) window used in previous studies (e.g. Black, 1988). The

3. The render may have noticed that the $\operatorname{Pr}(w)$ factor can be omitsed since it will not change the results of the maximization. It is included tere for expository convenience so that it is possible to compare results acnoss words with very different probabilitics. The factor also becomes important when an inomplete set of indicators is stored because of computational space corsirainis. Currently we assume a uniform prios probability for each Roget calegory $\left(\operatorname{Pr}\left(R_{\text {cal }}\right)\right)$, i.e. sense clnssification is based exclusively on contextual informution, independent of the underiying probubility of a given Roget category appenting at any point in the corpus. maximization over RCats is constrained to consider only those categories under which the polysemous word is listed, generally on the order of a half dozen or $\mathrm{so}^{4}$

For example the word crane appears 74 times in Groliers; 36 occurrences refer to the animal sense and 38 refer to the heavy machinery sense. The system correctly classified all but one of the machinery senses, yielding 99\% overall accuracy. The one misclassified case had a low score for all models, indicating a lack of confidence in any classification.

It is useful to look at one example in some more detail. Consider the following instance of crane and its context of \pm 10 words: ${ }^{5}$

lift water and to grind grain .PP Treadmills attached to cranes were used to lift heavy objects from Roman times,

The lable below shows the strongest indicators identified for the two categories in the sentence above. The model weights, as noted above, are equivalent to $\log \operatorname{Pr}(w \mid R C a t) / \operatorname{Pr}(w)$. Several indicators were found for the TOOLS/MACHINE class. There is very little evidence for the ANIMAL sense of crane, with the possible exception of water. The preponderance of evidence favors the former classification, which happens to be correct. The difference between the two total scores indicate strong confidence in the answer.

\begin{tabular}{|lc|ll|}
\hline TOOLS/MACH. & Weight & ANIMAL,INSECT & Weight \\
\hline lift & 2.44 & water & 0.76 \\
lift & 2.44 & & \\
grain & 1.68 & & \\
used & 1.32 & & \\
heavy & 1.28 & & \\
Treadmills & 1.16 & & \\
attached & 0.58 & & \\
grind & 0.29 & & \\
water & 0.11 & & \\
\hline TOTAL & 11.30 & TOTAL & 0.76 \\
\hline
\end{tabular}

4. Although it is often useful to restrict the search in this way, the restriction does someumes lead to trouble, especially when there are gaps in the thesaurus. For example, the category AMUSEMainT (" $876)$ lists a number of card playing tems, but for some reason, the word suit is no included in this list. As it happens, the Grolier's Encyclopedia contains 54 instances of the card-playing sense of swit, all of which are mislabeled if the search is limited to just those categories of suit that are listed in Roget's. However, if we open up the senrch to consider atl 1042 cniegories, then we find that all 54 instunces of suit are correctly labeled is AMUSEMENT, and moreover, the score is large in all 54 instances, indicating great confidence in the assignment. It is possible that the unrestricted search mode might be a good way to atlempt to fill in omissions in the thesuuns. In any cose, when suit is added to the AMUSEMENT catcgory, overall accuracy improves from $68 \%$ to $92 \%$.

5. This narower window is used for illustrative simplicity. 


\section{TABLE 1}

\begin{tabular}{|c|c|c|c|}
\hline Sense & Roget Category & $N$ & Corr. \\
\hline \multicolumn{4}{|c|}{ STAR (Hirst, 1987: N/A) } \\
\hline \multirow[t]{2}{*}{$\begin{array}{l}\text { Space Object } \\
\text { Celebrity } \\
\text { Star Shaped Object }\end{array}$} & $\begin{array}{l}\text { UNIVERSE } \\
\text { ENTERTAINIR } \\
\text { INSIGNIA }\end{array}$ & $\begin{array}{r}1422 \\
222 \\
56\end{array}$ & $\begin{array}{l}96 \% \\
95 \% \\
82 \%\end{array}$ \\
\hline & & 1700 & $96 \%$ \\
\hline \multicolumn{4}{|c|}{ MOLE (Hirst, 1987: N/A *) } \\
\hline $\begin{array}{l}\text { Quantity } \\
\text { Mammal } \\
\text { Skin Blemish } \\
\text { Digging Machine }\end{array}$ & $\begin{array}{l}\text { CHEMMCALS } \\
\text { ANIMAL,INSECT } \\
\text { DISEASH } \\
\text { SUPPOK'T }\end{array}$ & $\begin{array}{r}95 \\
46 \\
13 \\
4\end{array}$ & $\begin{array}{l}98 \% \\
100 \% \\
100 \% \\
100 \%\end{array}$ \\
\hline Digging Machine & & 160 & $99 \%$ \\
\hline \multicolumn{4}{|c|}{ GALLFY (Lesk, 1986: 50-70\% overall) } \\
\hline \multirow[t]{2}{*}{$\begin{array}{l}\text { Ancient Ship } \\
\text { Printer's Tray } \\
\text { Ship's Kjtchen }\end{array}$} & $\begin{array}{l}\text { SIIJP,BOAT } \\
\text { PRINIING } \\
\text { COOKING }\end{array}$ & $\begin{array}{r}35 \\
5 \\
2\end{array}$ & $\begin{array}{r}97 \% \\
100 \% \\
50 \%\end{array}$ \\
\hline & & 42 & $95 \%$ \\
\hline \multicolumn{4}{|c|}{ CONE (Lesk, 1986; 50-70\% oversll *) } \\
\hline \multirow[t]{2}{*}{$\begin{array}{l}\text { Part of Tree } \\
\text { Shape of Object } \\
\text { Part of Eye }\end{array}$} & $\begin{array}{l}\text { MANT } \\
\text { ANGULARTTY } \\
\text { VISION }\end{array}$ & $\begin{array}{l}71 \\
89 \\
13\end{array}$ & $\begin{array}{l}99 \% \\
61 \% \\
69 \%\end{array}$ \\
\hline & & 173 & $77 \%$ \\
\hline \multicolumn{4}{|c|}{ BASS (Hearst, 1991: 100\%; Speech Synthesis) } \\
\hline \multirow[t]{2}{*}{$\begin{array}{l}\text { Musical Senses } \\
\text { Fish }\end{array}$} & $\begin{array}{l}\text { MUSIC } \\
\text { ANIMAL,INSIECT }\end{array}$ & $\begin{array}{r}158 \\
69 \\
\end{array}$ & $\begin{array}{r}99 \% \\
100 \%\end{array}$ \\
\hline & & 227 & $99 \%$ \\
\hline \multicolumn{4}{|c|}{ BOW (Clear, 1989: < 67\%; Speech Synthesis) } \\
\hline $\begin{array}{l}\text { Weapon } \\
\text { Front of Ship } \\
\text { Violin Part } \\
\text { Ribbon } \\
\text { Bend in Object } \\
\text { Lowering Head }\end{array}$ & $\begin{array}{l}\text { ARMS } \\
\text { SHIP,BOAT } \\
\text { MUSICAI,INSTR } \\
\text { ORNAMINIATIION } \\
\text { CONVI:XITY } \\
\text { RESPICT }\end{array}$ & $\begin{array}{r}59 \\
34 \\
30 \\
4 \\
2 \\
0\end{array}$ & $\begin{array}{r}92 \% \\
94 \% \\
100 \% \\
25 \% \\
50 \% \\
\ldots\end{array}$ \\
\hline Lowering Head & & 129 & $91 \%$ \\
\hline \multicolumn{4}{|c|}{ TASTE (Clear, 1989: < 65\%) } \\
\hline \multirow[t]{2}{*}{$\begin{array}{l}\text { Preference } \\
\text { Flavor }\end{array}$} & $\begin{array}{l}\text { PARIJCUIARTIY } \\
\text { SENSATION }\end{array}$ & $\begin{array}{r}228 \\
80 \\
\end{array}$ & $\begin{array}{l}93 \% \\
93 \%\end{array}$ \\
\hline & & 308 & $93 \%$ \\
\hline \multicolumn{4}{|c|}{ INTEREST (Black, 1988: 72\%; Zernik, 1990: > 70\%) } \\
\hline \multirow{5}{*}{$\begin{array}{l}\text { Curiosity } \\
\text { Advantage } \\
\text { Financial } \\
\text { Share }\end{array}$} & REASONING & 359 & $88 \%$ \\
\hline & INJUSITCE & 163 & $34 \%$ \\
\hline & DEBT & 59 & $90 \%$ \\
\hline & PROPSETY & 21 & $38 \%$ \\
\hline & & 602 & $72 \%$ \\
\hline \multicolumn{4}{|c|}{ ISSUE (Zernik, 1990: < 70\%) } \\
\hline \multirow[t]{2}{*}{$\begin{array}{l}\text { Topic } \\
\text { Periodical } \\
\text { Stock }\end{array}$} & $\begin{array}{l}\text { POIJITCS } \\
\text { BOOKS,PERIODI } \\
\text { SLCURTIES }\end{array}$ & $\begin{array}{r}831 \\
28 \\
9\end{array}$ & $\begin{array}{r}94 \% \\
89 \% \\
100 \%\end{array}$ \\
\hline & & 868 & $94 \%$ \\
\hline
\end{tabular}

\begin{tabular}{|c|c|c|c|}
\hline Sense & Roget Category & $\mathbf{N}$ & Corr. \\
\hline \multicolumn{4}{|c|}{ DUTY (Gale et al, 1992: 96\%) } \\
\hline \multirow[t]{2}{*}{$\begin{array}{l}\text { Obligation } \\
\text { Tax }\end{array}$} & $\begin{array}{l}\text { DUTY } \\
\text { PRICE,FI: }\end{array}$ & $\begin{array}{r}347 \\
52\end{array}$ & $\begin{array}{l}96 \% \\
96 \%\end{array}$ \\
\hline & & 399 & $96 \%$ \\
\hline \multicolumn{4}{|c|}{ SENTENCF (Gale et al, 1992: 90\% *) } \\
\hline \multirow[t]{2}{*}{$\begin{array}{l}\text { Punishment } \\
\text { Set of Words }\end{array}$} & $\begin{array}{l}\text { IJGAL ACIION } \\
\text { GRAMMAR }\end{array}$ & $\begin{array}{l}128 \\
213\end{array}$ & $\begin{array}{l}99 \% \\
98 \%\end{array}$ \\
\hline & & 341 & $98 \%$ \\
\hline \multicolumn{4}{|c|}{ SLUG (Hirst, 1987: N/A *) } \\
\hline Animal & ANIMAL,INSI:CT & 24 & $100 \%$ \\
\hline Type Strip & PRINIING & 8 & $100 \%$ \\
\hline Mass Unit & WI:IGITI & 3 & $100 \%$ \\
\hline Fake Coin & MONEY & 2 & $50 \%$ \\
\hline Metallurgy & IMPULSE,IMPACI & 1 & $100 \%$ \\
\hline \multirow[t]{2}{*}{ Bullet } & ARMS & 1 & $100 \%$ \\
\hline & & 39 & $97 \%$ \\
\hline
\end{tabular}

Notes:

1) $N$ refers to the total number of each sense observed in the test corpus. Corr. indicates the percentage of those tagged correctly.

2) Because there is no independent ground truth to indicate which is the "conect" Roget category for a given word, the decision is a subjective judgement made by a single human judge, in this case the author.

3) As previously noted, the Roget index is incomplete. In four cases, identified by *, one missing category has been added to the list of possibilities for a word. These omissions in the lexicon have been identified as outlined in Footnote 4. Without these additions, overall system performance would decrease by $5 \%$.

4) Uses which an English speaker may consider a single sense are often realized by several Roget categories. For the purposes of succinel representation, such categories have been merged, and the name of the dominant category used in the table. As of this writing, the process has not been fully automated.

For many applications such as speech synthesis and assignment to an established dictionary sense number or possible French translations, this merging of Roget classes is not necessary.

The primary criterion for success is that words are partitioned into pure sense clusters. Words having a different sense from the majority sense of a partition are graded as errors.

5) Examples with the anmotation 'speech synthesis' have multiple pronunciations corresponding to sense distinctions. Their disambiguation is important in speech processing.

6) All results are based on $100 \%$ recall. 


\section{Evaluation}

The algorithm described above was applied to 12 polysemous words previously discussed in the sense disambiguation literature. Table 1 (previous page) shows the system's performance. Authors who have discussed these words are listed in parentheses, along with the reported accuracy of their systems. Direct comparisons of performance between researchers is difficult, compounded by variances in corpora and grading criteria; using the same words is an attempt to minimize these differences.

Regrettably, most authors have reported their results in qualitative terms. The exceptions include Zernik (1990) who cited "recall and precision of over 70\%" for one word (interest) and observed that results for other words, including issue, were "less positive." Clear (1989) reported results for two words (65\% and 67\%), apparently at $85 \%$ recall. Lesk (1986) claimed overall "50-70\%" accuracies, although it is unclear under which parameters and constraints. In a 5 word test set, Black (1988) observed $75 \%$ mean accuracy using his optimal method on high entropy, 4-way sense distinctions. Hearst (1991) achieved $84 \%$ on simpler 2-way distinctions, editing out additional senses from the test set. Gale, Church and Yarowsky (1992) reported 92\% accuracy, also on 2-way distinctions.

Our current work compares favorably with these results, with $92 \%$ accuracy on a mean 3-way sense distinction ${ }^{6}$. The performance is especially promising given that no hand tagging or special corpora were required in training, unlike all other systems considered.

\section{Limitations of the Method}

The procedure described here is based on broad context models. It performs best on words with senses which can be distinguished by their broad context. These are most typically concrete nouns. Performance is weaker on the following:

Topic Independent Distinctions: One of the reasons that interest is disambiguated poorly is that it can appear in almost any context. While its "curiosity" scnse is often indicated by the presence of an academic subject or hobbie, the "advantage" sense (to be in one's interests) has few topic constraints. Distinguishing between two such abstractions is difficult. ${ }^{7}$ However, the financial

6. Thix result is a fair measure of performance on words used in previous studies, and may be useful for comparison across systems. However, as wonds previously discussed in the literature may not be representutive of typical English polysemy, mean perfornance on a completely random ses of words should differ.

7. Black (1988) has noted that this distinction for interest is strongly correlated with the plurality of the word, a feature we cumently don't utilize. sense of interest is readily identifiable, and can be distinguished from the non-financial uses with $92 \%$ accuracy. Other distinctions between topic independent and topic consurained senses appear successful as well (c.g. taste, issue. duty and sentence).

Minor Sense Distinctions within a Category: Distinctions between the medicinal and narcotic senses of drug are not captured by the system because they both belong to the same Roget category (REMEDY). Similar problems occur with the musical senses of bass. Roget's Thesaurus offers a rich sub-hierarchy within each category, however. Future implementations will likely use this information, which is currently ignored.

Verbs: Verbs have not been considered in this particular study, and it appears that they may benefit from more local models of their typical arguments. The unmodified system does seem to perform well on verbs which show clear topic distinctions such as fire. It's weapon, engine, furnace, employee, imagination and pottery senses have been disambiguated with $85 \%$ accuracy.

Pre-Nominal Modifiers: The disambiguation of prenominal modifiers (adjectives and compound nominals) is heavily dependent on the noun modified, and much less so on distant context. While class-based Bayesian discrimination may be useful here as well, the optimal window size is much narrower.

Idioms: These broad context, topic-based discriminators are also less successful in dealing with a word like hand, which is usually found in fixed expressions such as on the other hand and close at hand. These fixed expressions have more function than content, and therefore, they do not lend themselves to a method that depends on differences in content. The situation is far from hopeless, as many idioms are listed directly in Roget's Thesaurus and can be associated with a category through simple table lookup. Other research, such as Smadja and McKeown (1990), have shown more general ways of identifying and handling these fixed expressions and collocations.

Given the broad set of issues involved in sense disambiguation, it is reasonable to use several specialized tools in cooperation. We already handle part of speech distinctions through other methods; an efficient idiom recognizer would be an appropriate addition as well.

\section{Linking Roget Categories with other Sense Representations}

The Roget category names tend to be highly mnemonic and may well suffice as sense tags. However, one may want to link the Roget tags with an established reference such as the sense numbers one finds in a dictionary. We accomplish this by applying the models described above to the text of the definitions in a dictionary, creating a table of correspondences between Roget categories and 
sense numbers. Results for the word crane arc illustrated below for two dictionaries: (1) COBUILD (Sinclair, 1987), and (2) Collins English Dictionary, First Edition (CED1) (Hanks, 1979).

\begin{tabular}{|c|c|c|}
\hline RCAT & Sense \# & Definition \\
\hline TOOLS & crane 1.1 & a machine with a long movable \\
\hline ANIMAL & crane 1.2 & large bird with a long neck and \\
\hline ANIMAL & crane 1 & any large long-necked long-leg \\
\hline ANIMAL & crane 2 & any similar bird, such as a her \\
\hline TOOLS & crane $?$ & a device for lifting and moving \\
\hline ToOLS & crane 4 & a large urolley carrying a boom \\
\hline
\end{tabular}

It may also be possible to link Roget category tags with "natural" sense tags, such as translations in a foreign language. We use a word-aligned parallel bilingual corpus such as the French-English Canadian Hansards for this purpose. For example, consider the polysemous word duty which can be translated into French as devoir or droit, depending on the sense (obligation or tax, respectively). When the Grolier-trained models are applied to the English side of the Hansards, the words tagged PRICE,FIE most commonly aligned with the French words droits (256), droit (96) and douane (67). Words labeled DUTY (the Roget category for Obligation) most frequently aligned with devoir (205). These correlations may have useful implications for machine Iranslation and bilingual lexicography.

\section{Other Sense Disambiguation Methods: The Knowledge Acquisition Bottleneck}

Word sense disambiguation is a long-standing problem in computational linguistics (Kaplan, 1950 ; Yngve, 1955; Bar-Hillel, 1960), with important implications for a variety of practical applications including specch synthesis, information retrieval, and machine translation. Most approaches may be characterized by the following generalizations: 1) They tend to focus on the search for sets of word-specific features or indicators (typically words in context) which can disambiguate the senses of a word. 2) Efforts to acquire these indicators have faced a knowledge acquisition bottencek, characterized by either substantial human involvement for each word, and/or incomplete vocabulary coverage.

The AI community has enjoyed some success handcoding detailed "word experts" (Small and Rieger, 1982; Hirst, 1987), but this labor intensive process has severely limited coverage beyond small vocabularies.

Others such as Lesk (1986), Walker (1987), Veronis and Ide (1990), and Guthrie et al. (1991) have tumed to machine readable dictionaries (MRD's) in an effort to achieve broad vocabulary coverage. MRD's have the useful property that some indicative words for each sense are directly available in numbered definitions and examples. However, definitions are often too short to provide an adequate set of indicators, and those worls which are found lack significance weights to identify which are crucial and which are merely chaff. Dictionaries provide well structured but incomplete information.

Recently, many have turned to text corpora to broaden the range and volume of available examples. Unlike dictionaries, however, raw corpora do not indicate which sense of a word occurs at a given instance. Several researchers (Kelly and Stone, 1975; Black, 1988) have overcome this through hand tagging of training examples, and were able to discover uscful discriminatory patterns from the partitioned contexts. This also has proved labor intensive. Others (Weiss, 1973; Zernik, 1990; Hearst, 1991) have attempted to partially automate the handtagging process through bootstrapping. Yet this has still required significant human intervention for each word in the vocabulary.

Brown et al. (1991), Dagan (1991), and Gale et al. (1992) have looked to parallel bilingual corpora to further automate training set acquisition. By identifying word correspondences in a bilingual text such as the Canadian Parliamentary Proceedings (Hansards), the translations found for each English word may serve as sense tags. For example, the senses of sentence may be identified through their correspondence in the French to phrase (grammatical sentence) or peine (legal sentence). While this method has been used successfully on a portion of the vocabulary, its coverage is also limited. Currently available bilingual corpora lack size or diversity: over half of the words considered in this study either never appear in the Hansards or lack examples of secondary senses. More fundamentally, many words are mutually ambiguous across languages. French would be of little use in disambiguating the word interest, as all major senses translate as intérêt. More promising is a non-Indo European language such as Japanese, which should avoid such mutual ambiguity for etymological reasons. Until more diverse, large bilingual corpora become available, the coverage of these methods will remain limited.

Each of these approaches have faced a fundamental obstacle: word sense is an abstract concept that is not identified in natural text. Hence any system which hopes to acquire discriminators for specific senses of a word will need to isolate samples of those senses. While this process has been partially automated, it appears to require substantial human intervention to handle an unrestricted vocabulary.

\section{Conclusion}

This paper has described an approach to word sense disambiguation using statistical models of word classes. This method overcomes the knowledge acquisition bottleneck faced by word-specific sense discriminators. By entirely circumventing the issue of polysemy 
resolution in training material acquisition, the system has acquired an extensive set of sense discriminators from unrestricted monolingual texts without human intervention. Class models also offer the additional advantages of smaller model storage requirements and increased implementation efficiency due to reduced dimensionality. Also, they can correclly identify a word sense which occurs rarely or only once in the corpus performance unatainable by statistically trained wordspecific models. These advances are not without cost, as class-based models have diluted discriminating power and may not capture highly indicative collocations specific to only one word. Despite the inherent handicaps, the system performs better than several previous approaches, based on a direct comparison of results for the same words.

\section{Acknowledgements}

Special thanks are due to Ken Church and Barbara Grosz for their invaluable help in restructuring this paper, and to Bill Gale for the theoretical foundations on which this work rests. The author is also grateful to Marti Hearst, Fernando Pereira, Donald Hindle, Richard Sproat, and Michael Riley for their comments and suggestions.

\section{References}

Bar-Hillel (1960), "Automatic Translation of Languages," in Advances in Computers, Donald Booth and R. E. Meagher, eds., Academic, New York.

Bluck, Frsa (1988). "An Experiment in Compulational Discrimination of English Word Sences," IBM Jowrnal of Research and Development, v 32, pp 185-194.

Brown, Peter, Stephen Delle Pietra, Vincent Delta Pietra, and Robert Meroer (1991), "Word Sense Disumbiguntion using Statistical Methods," Proceedings of the 29th Annual Meeting of the Association for Computational Linguistics, pp 264-270.

Brown, Peter, Vincent Dellı Pietra, Peter deSouz», and Robert Mercer (1990), "Clant-based n-gram Models of Natural Langunge," Proceedings of the IBM Natural Language ITL, Paris, France, pp 283. 298.

Chupnan, Roben (1977). Roget's Iniernational Thesaurus (Fourth Edition), Harper and Row, Now York.

Choucka, Yascov, and Serge Lusignam (1985), "Disambiguation by Short Contexts," Compusers and the Hunanities, v 19. pp. 147-158.

Church, Keaneth (1989). "A Stochastic Parts Program an Noun Phrase Parser for Unrestricted Text," Proceeding, IEEE Internalionat Conference on Acoustics, Speech and Signal Processing. Glssgow.

Clear, Jeremy (1989). "An Experiment in Automatic Word Sense Identification," Intemal Document, Oxford Universily Press, Oxford.

Courell, Gurrison (1989). A Connectionits Approach to Word Sense Disambiguation, Pitman, London.

Dagen, Ido, Alon Itai, and Ulrike Schwall (1991), "Two Languager are more Informative then Ore," Proceedings of the 29th Annalal Meeting of the Assaciation for Compulational Linguistics, pp 130-137.

Gale, Williem, Kenneth Church, and David Yarowsky (1992), "Divcrimination Deciaions for 100,000-Dimensional Spaces" AT\&T Statistical Research Report No. 103.

Gake, William, Kenneth Church, and David Yarowsky (1992), "A
Method for Disumbiguzting Word Senses in a Large Corpus," to appear in Computers and Humanities.

Granger, Richard (1977), "FOUL-UP A program that figures out meanings of words from contex1," IJCAII-77. pp. 172-178.

Guthrie, J., L. Guthric, Y. Wilks, and H. Aidinejad (1991), "SubjeaDependent Co-occurrence and Word Sense Disumbiguation," Procecdings of the 29th Annual Mecting of the Association for Computational Linguistics, pp 146-152.

Hunks, Patrick (ed.) (1979), Collins English Dictionary, Collins, London and Glasgow.

Hearst, Marti (1991), "Noun Homograph Dismmbiguation Using Local Context in Lurge Text Corpora," Using Corpora, Univentity of Waterlow, Waterloo, Onterio.

Hirst, Graeme. (1987), Semantic Interpretation and the Resolution of Ambiguity, Cambridge Universily Press, Cambridge.

Kaplan, Abraham (1950), "An Experimental Study of Ambiguity in Context," cited in Mechanical Translation, v. 1, nos 1-3.

Kelly. Edwund, and Phillip Stone (1975), Compuser Recognition of English Word Senses, North-Holland, Amsterdam.

lesk, Michael (1986), "Automatic Sense Disambiguation: How to tell a Pine Come from an Ice Cream Cone," Proceeding of the 1986 SIGDOC Conference, Associution for Computing Mechinery, New York.

Miller, George (1990), "Wordnel: An On-line Lexical Databuse," International Journal of Lexicography, 4(3), 1990. (Special Issue).

Mostcller, Fredrick, and David Wallace (1964), Inference and Disputed Authorship: The Federalist, Addison-Wesley, Reading, Massachusets.

Salton, G, (1989), Awomatic Text Processing, Addison-Wesley Publishing Co.

Sindair, J., Hanks, P., Fox, G., Moon, R., Swock, P. et sl. (eds.) (1987) Collins Cobuild English Language Dictionary, Collins, London and Glasgow.

Slator, Brian (1991), "Using Context for Sense Preference," in Zemik (cd.) Lexical Acquisition: Exploiting On-Line Resources to Build a Lexicon, Lewrence Erlbuum, Hillsdale, NJ.

Smadja, F. and K. McKcown (1990), "Automatically Exiracting and Representing Collocations for Language Generation," Proceedings of the 28th Annual Mecling of the Association for Computational Linguistics.

Small, S, and C. Rieger (1982), "Parsing and Conuprehending with Wond Experts (A Thoory and its Realization)," in Strategies for Natural Language Processing. W. Lehnert and M. Ringle, eds., Lawrence Erlbeum A ssociates, Hillsdale, NJ.

Walker, Donald (1987), "Knowledge Resource Tools for Accessing Large Text Files," in Machine Translation: Theorelical and Methodological Issues, Sergei Nirenberg, ed. Cambridge University Press, Cambridge, Fngland.

Weiss, Stephen (1973), "Learning to Disambiguate," Information Storage and Retriewal, v. 9, pp 33-41.

Veronis, Jean and Nancy Ide (1990), "Word Sense Disambiguation wilh Very Large Neural Networks Extracted from Machine Readuble Dictionn ries," in Proceedings COLING-90, pp 389-394.

Yngve, Victor (1955). "Syntax and the Problem of Multiple Meaning," in Machine Translation of Languages, Wiltiam Locke and Donald Booth, eds., Wiley, New York.

Zernik, Uri (1990) "Tagging Word Senses in a Corpus: The Needle in the Haystack Revisited," in Text-Based Intelligent Systems: Current Research in Texi Analysis, Information Extracition, and Retrieval, P.S. Jacobs, ed., GE Research \& Development Center, Schenectudy, New York. 